FINDING A MAXIMUM-GENUS GRAPH IMBEDDING

- Merrick L. Furst, Jonathan L. Gross, \& Lyle A. McGeoch

CUCS $-192-85$ 


\title{
Finding a Maximum-Genus Graph Imbedding
}

\author{
December 2, 1085
}

Merrick L. Furst ${ }^{1}$ Jonnthan L. Gro.4s ${ }^{2}$ Lyle A. McGeoch ${ }^{1}$

\section{Abstract}

The computatioual complexity of coustructing the imbeddings of a given graph into surfaces of different genus is not well-understood. In this paper, topologiciul methods and a reduction to lincar matroid parity are used to devclop a polynomial-time algorithm to find a maximum-genus cellular imbedding. This secens to be the first imbeddling algorithm for which the rmuning time is not exponential in the genus of the imberding surface.

\section{Introduction}

Lower-dimensional topology has long been approached combinatorially. For most questions about imbeddings, there exist exhaustive algorithms. Since the number of combinatorial equivalence classes of graph inbeddings is a super-exponential function of the number of vertices, such exhaustive algorithms are computationally infeasible.

There have been several algorithinic achievenients. Hopcroft and Tarjan [ITT obtained a linear-time algorithm to test planarity of graphs, while Gross and Rosen [GR] showed how to test planarity of 2-complexcs. Filotti $[F]$ found a polynomial-tine algorithm to determine if a cubic graph can be imbedded in the torus, and Filotti, Miller, and Reif [FMR] generalized this work with an algorithm to imbed a graph in a surface of minimum genus $G$ in timc $O\left(v^{O}(G)\right)$. All these algorithms are based on extending partially imbedded graphs, and they all produce an

1 Computer Science Department, Carnegic-Mellon University, Pittsburgh, PA 15213.

2 Computer Science Department. Columbia University, New York, NY 10027. Research supported in part by NSF grant MCS-8308805, in part by Presidential Young Investigator grant DCR-8352081, in part by ONR Contract N00014-85-0768, and in part by an NSF Graduate Fellowship. 
inubcylding whonever it exists. Raif [R.7!)] showed that there are limits to this alpproukh by proving that the problen of deciding whether a partial imbedding in somes surface caun be: extended to a full imberlding in tlat surfare is NP-romplete.

Our present concern is the determination of the "maximum genus" of a graph. There is no limit to the unmber of haulles one might ald to a surfare in which a graph is alreidly imberlded. For the concept of mibximun gemus to be: meimingrul, one must stipulate that every region of the imberlding be cellular - that. is, the interior of the region must. be homeomorphic to an open disk. Such a restriction is in no way artilicial. It corresponds to restricting handles to be "coscntial" .

Maximum-genus imbeddings, and the relatcd notion of upper-imbeddable graphs, have reccived considerable attention in recent ycars. A graph is called upper-imbeddable if it has a maxinumn-genus imberlding with one or t.wo fizces. Nordhauy, Stewart, and White [NSW], Ringeisen [R70,R72], and Zaks [Z] showed that. various classes of griphy were upper-imbeddable. Nebeský [N] and Jungerman [J] described combinatorial invariants of upper-imbedelable graphs. Xuong [X79b] showed that all graphs with two disjoint spanning trees, such as 4-edge connected graphs, are upper-imbeddable.

We consider the computational complexity of obtaining a maxinum-genus imbedrling. Our starting point is the combinatorial characterization by Xuong [X79a] of the maximum genus of a graph. This involves the consideration of all spanning trecs of a grapl, of which there can be exponentially many. We improve the obvious exponential-time algorithm to a polynomial-time algorithm.

\section{Preliminaries about Topological Graph Theory}

In topological graph theory, a "graph" is defined to be a (possibly) non-simplicial 1-complex. In other words, multiple adjacencies and self-loops are permitted. There are many reasons for this generality. In particular, the most powerful presently-known way to construct an imbedding of a large simplicial graph into a large-genus surface is to derive it as a branched covering of an imbedding of a smaller, non-simplicial graph - ideally with one vertex and many self-loops - into a smaller-genus surface. (See Gross and Tucker [GT] or White [W].)

In this paper, we consider only simplicial (simple) graphs: those without self-loops or multiple adjacencies. Any graph containing self-loops and multiple adjacencies can be transformed into a simplicial graph by inserting one or more vertices in the intcrior of these edges. Moreover, the resulting graph is homeomorphic to the original graph, and accordingly, it has the same maximum genus. This enables us to simplify the notation. 


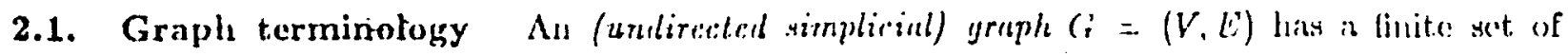

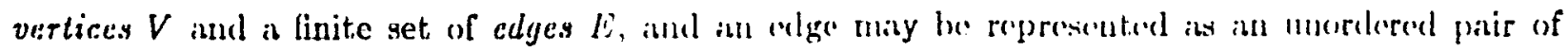
vertices $(v, w)$, i.e. its endpoints. An wlge is saicl lo be incident on its collpoinls. Two distinct colgey are saidi to be adjacent if they are incielent on ib common vertex.

The degree of a vertex is the number of edges incidcut on it. $\Lambda$ path from vertex $w$ tos vertex $w$ is a stequenre of colges $\left(v, u_{1}\right),\left(u_{1}, u_{2}\right), \ldots,\left(u_{k}, v\right)$ in $E$, such that the vortices $v, u_{1}, u_{2}, \ldots, u_{k}$, and $w$ are distinct. IIowever, if the starting point $v$ is the same aw the linial point $w$, the seculuence is called a cyule. A graph is connectel if there is a path betwcen every pair of its vertices. $\Lambda$ connected, acyclic graph is called a tree. By a spanning tree of graph (r, we mean a subgraph that is a tree and contains cvery vertex.

The notation $G+e$ is au abbreviation for the graph $(V, E \cup\{e\})$, and the uotation $G-e$ is an abbreviation for the graph $(V, E-\{e\})$.

A graph is directed if cach celge is thought to have a beginning and an end. We represent a directed edge as an ordered pair. Unless it is otherwise obvious from context, the graphs discussed will be connected and undirected.

2.2. Surfaces Our terminology is compatible with that of Gross and Tucker [GT] and of White [W].

The topological spaces of interest here are all horncomorplic to subspaces of $E^{3}$. A homeomorphism between two topological spaces is a continuous bijcctive mapping with a continuous inverse. A connected topological space is a surface if cvery point has a neighborhood that is lomcomorphic to the closed unit disk. A surface $S$ is orientable if it does not contain a Möbius band.

We deal only with closed orientable surfaces. Every such surface $S$ is homeomorphic to a generalized torus. The number of handles is denoted $\gamma(S)$ and is called the genus of the surface. A sphere, for cxample, is a surface of genus 0 , a torus is a surface of genus 1 , and a 2-handled toris is a surface of genus 2.

\subsection{Graph imbeddings and faces Although a graph is an abstract combinatorial object,} there is a topological representation of it: in Euclidean 3-space, we represent each vertex by a distinct point and each edge by a distinct curve between the two endpoints, where a curve means a homeomorphic image of the unit interval $[0,1]$. We require that the interior of an edge intersect no other edge or vertex of the graph. When referring to a graph in a topological setting, we mean such a representation. 


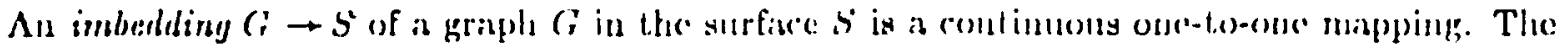
components of $S-G$ are called rengion.s. If call engion is homeomorphic: to an open disk, the imbedcling is cellulur, and the: regions are called fores.s. All our imbedelings are collular. The set of farces of in imbeddling is denoted $F$.

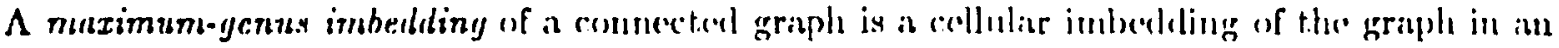
orientiables surface haviug maximum gonus anong all such imberldiug surfaces. The: Puler polyherlrad equation

$$
|V|-|E|+|F|=2-2 \gamma(S)
$$

bolds for all celludar imbruldingy. Thus, a maximum-genus inberding is the same thing as a minimuun-faceconnt imbedding.

2.4. Rotation systems A rotation at a vertex $v$ is a cyclic permutation of the exlges incident on it. Since our graphs arc simplicial, we may specify a rotation at $v$ in the format

$$
\text { v. } u_{1} u_{2} \ldots u_{d}
$$

where the vertices $u_{1}, \ldots, u_{d}$ are the opposite endpoints of the edges incident on $v$. It follows that a vertex $v$ with degree $d$ admits $(d-1)$ ! different rotations.

A list of rotations, one for each vertex, is call a rotation system for the graph. This concrpt is due to Heffter $[\mathrm{H}]$. Starting with a graph imbedding in an oriented surface, there corresponds an obvious rotation system, namely, the one in which the rotation at each vertex is consistent with the cyclic order of the neighboring vertices in that imbedding.

Edmonds $[E]$ was first to call attention explicitly to a method for inverting that correspondence. To each oricnted edge $(u, v)$, one assigns the oriented edge $(v, w)$ such that vertex $w$ is the immediate successor of vertex $u$ in the rotation at vertex $v$. The result is a permutation on the set of oriented edges, that is, on the set in which each undirected edge appears twice, once with each possible direction. In each edge-orbit under this permutation, the consecutive oriented edges line up head to tail, from which it follows that they form a directed cycle in the graph. We observe that it is possible for both orientations of the sarne edge to appear twice in the same edgc-orbit. If there are $n$ oriented edges in the orbit, then an $n$-sided polygon can be fitted into it. Fitting a polygon to every such edge-orbit results in a polygon on both sides of each edge, and collectively the polygons form a surface in which the graph is cellularly imbedded.

Sometimes one describes the rotation system of a graph pictorially, as in Figure 2.1. The graph is drawn in the plane so that the incidence of edges at each vertex is consistent with the rotation 


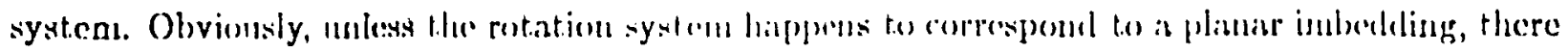

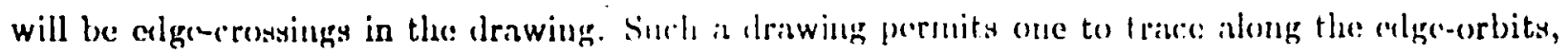

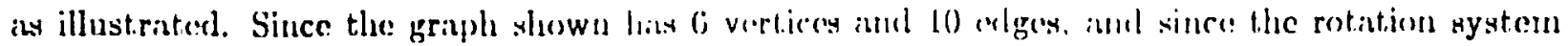
haw 2 edge-orbits, the inbedding surfare hiss bulder dharacteristic $6-10+2$, which equals -2 , from which it follows that. the imbedliug surfiure has genus two.

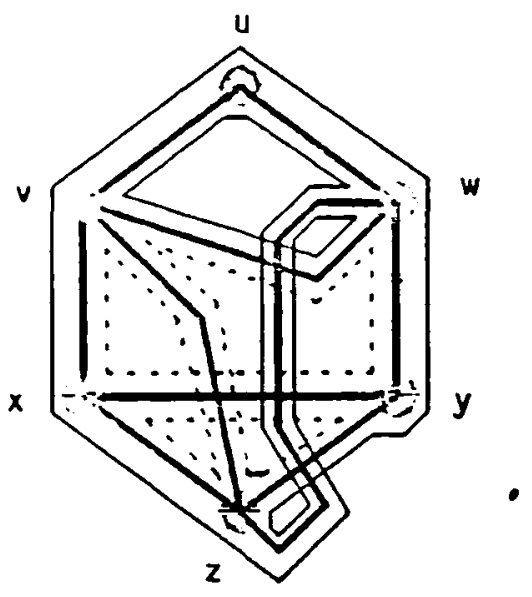

Figure 2.1. A graph with two edge-orbits in its rotation system.

The existence of the bijcctive correspondence between the cellular imbeddings of a graph and the rotation systems enables us to reformulate the problem of finding the maximum genus of the graph as a problem of finding a rotation system with the mininum number of edge-orbits. Since edge-orbits correspond to boundary-walks of faces, this is equivalent to secking a ininimumfacecount imbedding.

We can depict the boundary-walk of each face of an imbedding as a directed graph with one directed edge for each traversal of the undcrlying undirected edge, and multiple copies of each vertex; the boundary-walks for the rotation system of Figure 2.1 are shown in Figure 2.2. Any closed boundary-walk can also be written as an alternating (cyclic) sequence of vertices and edgcs $v_{1} e_{1} v_{2} e_{2} \ldots e_{k} v_{1}$. A subsequence $e_{i} v e_{i+1}$ of a walk is callell a corner, corresponding in an obvious way to the geometric corner of a face of a polygonal imbedding.

2.5. Adding and deleting edges If an edge is added to, or deleted from, an imbedded graph, then all faces in the imbedding are unchinged except those incident on that edge. Furthermore, either two faces are merged or one face is split into two faces. 

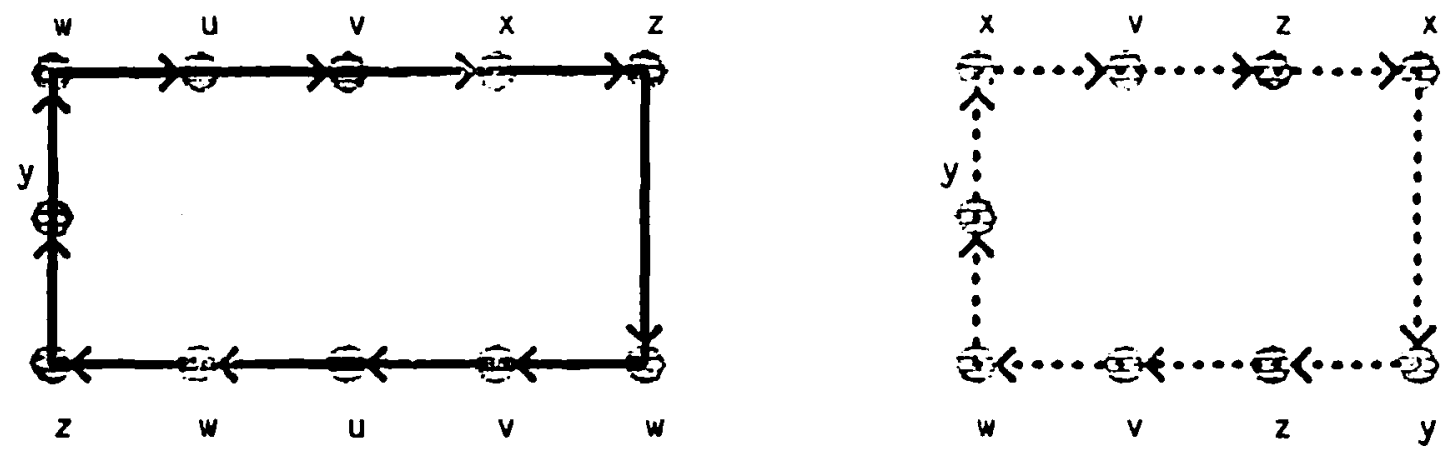

Fignare 2.2. The boundary-walks of the imbeddin! in Figure 2.1 .

Suppose that an colge $e=(u, v)$ is iulded to a graph and its imbedding, so that its cends are inserted between two corners of one face. If the boundary-walk around the original face was of the form $v \alpha_{1} w \alpha_{2} v$, where $\alpha_{i}$ is a subwalk, then as illustrated hy Figure 2.3, the new edge splits the old boundary-walk into two wilks: $v \alpha_{1} w e_{1} v$ and $w \alpha_{2} v e_{2} w$. Similarly, if an edge $e$ that is common to two faces is deleted from au imbedding, then two boundary-walks are merged and the new imbedding has one less face.

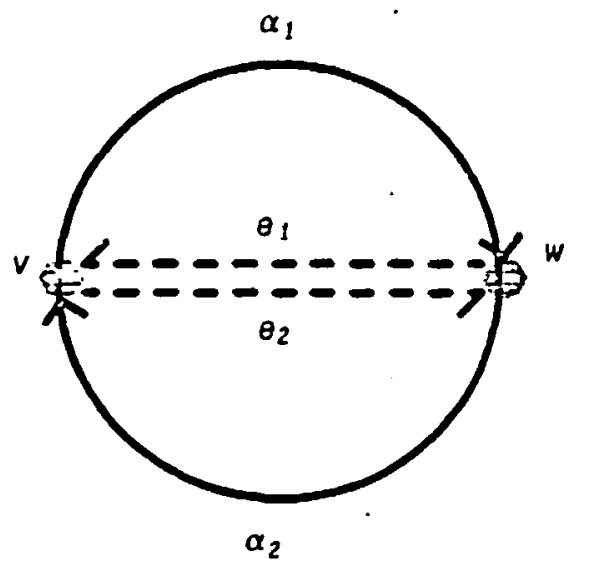

Figure 2.3. Adding an edge across a face.

If an edge is added to a graph and its ends are inserted between corners of two different faces, then both those faces are merged into one larger face. In particular, suppose that new edge $e$ runs from the corner of $v$ in boundary-walk $v \alpha_{1} v$ to the corner of $w$ in boundary-walk $w \alpha_{2} w$. Then a merged face results, with boundary-walk $v e_{1} w \alpha_{2} w e_{2} \alpha_{1} v$, as depicted in Figurc 2.4. Likewise, the deletion of an edge $e$ occurring twice on one boundary-walk splits the corresponding face into two 


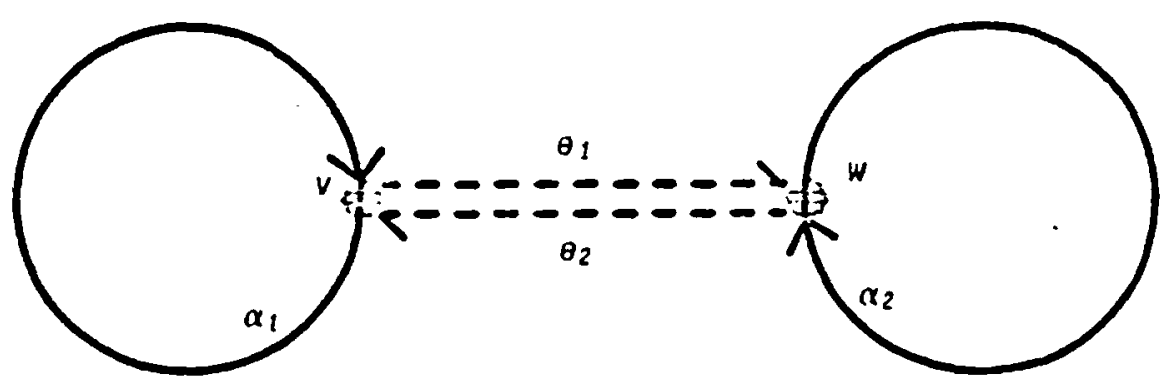

Fignre 2.4. Adling an edge between two faces.

sunnller faces.

\section{Maximum-Genus Imbeddings}

We now direct our attention to the problem of constructing a maximum-genus imbedding. Xuong [X79a] proved that calculating the maximum genus of a graph is reducible to calculating the value of a combinatorial invariant which he called its deficiency.

The deficiency $\xi(G, T)$ of a spanning tree $T$ in a graph $G$ is defined to be the number of connected components of $G-T$ that contain an odd number of edges. The deficiency $\xi(G)$ of a graph $G$ is defined to be the minimum trce deficiency over all spanning trees $T$ of $G$. We call a spanuing tree that realizes $\xi(G)$ a $X$ uong tree. Figure 3.1 shows a graph and one of its Xuong trees. Since the complement of the Xuong trec has two odd components, it follows that the graph has deficiency two.

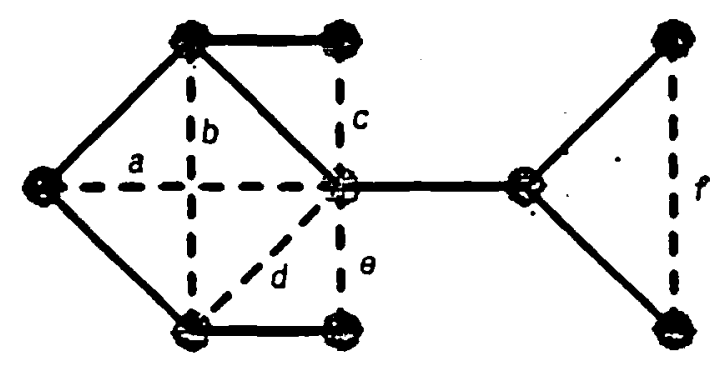

Figure 3.1. A spanning tree (solid edges) with minimum deficiency. 


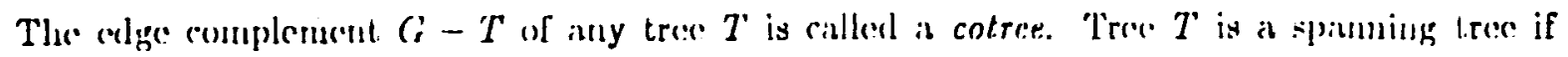

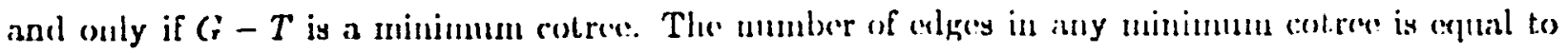

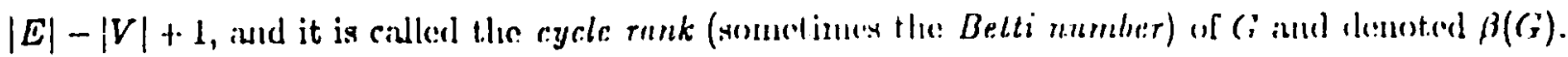

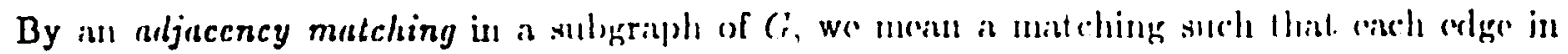
the subgraph is paired with an adjacont colge. For example, one maximum inljarency matching in

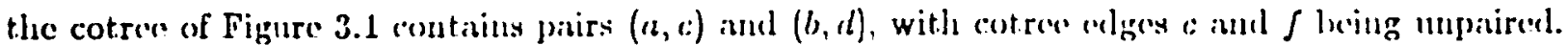

The: following reorganization of Xnong's methods and rederivation of his results is needed for our coustruction of a maxinum-gonus algorithm.

LEMMA 3.1. If a graph (; has a complrtely-patitell minimum cotree, then $G$ has a one-face imbedding.

Proof. By induction on $k$, the number of edge pairs in the minimum cotree.

BASE CASE: $k=0$. In this care the graph $G$ is a tree, and cvery imbedding lias exactly one face.

INDUC'TIVE CASE: $k>0$. As an induction hypothesis, assume that a graph with $k-1$ pairs of edges in a minimum cotrce has a one-face imbedding. We now arguc that we can add a new pair of adjacent edgey $e=(v, w)$ and $f=(w, x)$ to the onc-face imbedded graph in the following manner. First insert edge $e$ into the one face in any way between vertices $v$ and $w$, thercby splitting the single face in two. Note that vertex $w$ now has corncrs on both faces. Then insert cdge $f$ between some corner of $x$ and a corner of $w$ that lies on a different face (see Figure 3.2). This merges the two faces, thereby resulting in a one-face imbedding of $G+e+f$.

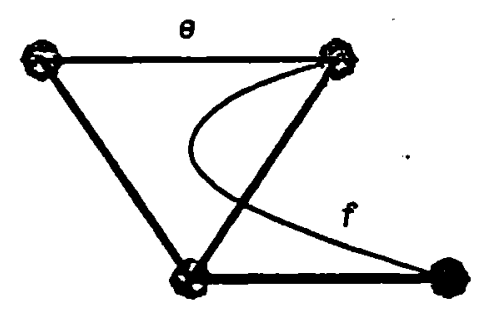

Figure 3.2. Adding adjacent edges $e$ and $f$ to a one-face imbedding.

LEMMA 3.2. If a graph $G$ has a minimum cotree with $k$ unpaired edges, then $G$ has an imbedding with at most $k+1$ faces. 


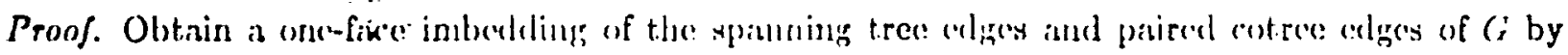

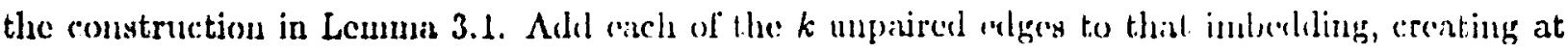
most one new face for cach chlge.

Lommas 3.1 aud 3.2 are constructive, and griven a maximum anjacency malching for a minimum cotroe, any reasomable implementialion of the construction will rmu in polynomial-time. A naive upper bound on the running time for a graphl with e chlges is $O\left(r^{2}\right)$.

LliMMA 3.3. If a graph $G$ has a one-face imbedling, then it has a completely-puired mirimum cotree.

Proof. By induction on the number of edges, $k$, in $G$.

BAsE CAsE I: $k=|V|-1$. In this case, the graph $G$ is a spanning tree for itself, the cotree is empty, and trivially all edges arc paired.

BASE CASE II: $k=|V|$. In this casc, the graph $G$ is a spanning trce plus one extra edgc. A spanning tree can ouly be imbedded with one face, and the addlition of the extra edge to such a one-face imbedding must break the face in two. Thus, the graph $G$ can only be inbedded with two faces, and the lemma holds vacuously.

INDUCTIVE CASE I: $k>|V|$, and $G$ has a vertex $v$ of degree one. Consider the graph $G^{\prime}$ obtained by deleting $v$ and its incident edge $e=(v, w)$ from $G$. Since $G$ has as one-face imbedding, we can readily construct a onc-face imbedding of $G^{\prime}$ by starting with the one-face imbedding for $G$ and deleting $e$ and $v$. By induction hypothesis, the graph $G^{\prime}$ has a minimum cotree $C$ with all its edgcs paired. Since the edge $e$ must be in any spanning tree of $G, C$ is a completely-paired minimum cotree of $G$.

INDUCTIVE CASE II: $k>|V|$, and $G$ has no vertex of degree one. Consider the boundarywalk around the single face. There must be an edge $r=(u, v)$ whose two appearances in the walk occur as closely together as the two appearances of any other edge. Give the two appearances of $r$ the labels $\vec{r}$ and $\leftarrow$, so as to minimize the length of subwalk $\alpha$ from $\vec{r}$ to $\overleftarrow{r}$. Subwalk $\alpha$ must contain of at least one edge other than $r$, or else $G$ would have a vertex of degree one, a contradiction. Similarly, if $\vec{s}$ is the edge following $\vec{r}$, then $\alpha$ can not also contain $\overleftarrow{s}$, since the two appearances of edge $s$ would then be closer together than those of edge $r$. Therefore, the boundary-walk around $G$ 's face must be of the form $u \vec{r} v \vec{s} w \alpha_{1} v \overleftarrow{r} u \alpha_{2} w \overleftrightarrow{s} v \alpha_{3} u$, where $s=(v, w)$ is an edge adjacent to $r$ in $G$, and $\alpha_{1}, \alpha_{2}$, and $\alpha_{3}$ are subwalks. See Figure 3.3.

Delete edges $r$ and $s$ from $G$ to obtain the graph $G^{\prime}$. Vertices $u$ and $v$ are connected in $G^{\prime}$ by edges that appeared in subwalk $\alpha_{3}$, and vertices $v$ and $w$ are connected by edges that appeared in 


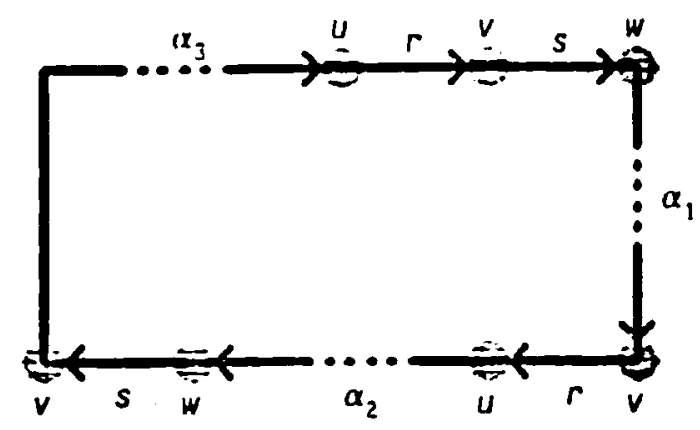

Figurc 3.3. Boundary-wilk of $G$ before deleting edgey $r$ and $s$.

subwalk $\alpha_{1}$. Every other vertex in $C^{\prime \prime}$ apperared in $\alpha_{1}, \alpha_{2}$ or $\alpha_{3}$ and is thus connected to $u, v$, or $w$ by edges in ( ${ }^{\prime}$. Since those three vertices are all connected, it follows that $G^{\prime}$ is connected.

By the induction hypothesis $G^{\prime}$ his a cotree $C$ that is completely paired. Clearly the tree $G^{\prime}-C$ is also a spanning trec of $G$. Elgey $r$ and $s$ can be paired and added to $C$ to form a completely-paircd minimum cotrec of $G$.

LEMMA 3.4. If a graph $G$ has a $(k+1)$-face imbedling, then it has a minimum cotree with at most $k$ unpaired edges in its maximum adjacency matching.

Proof. By induction on the number $k$.

BASE CASE: $k=0$. This follows from the previous lemma.

INDUCTIVE CASE: $k>1$. Let $e$ be an edge in $G$ that lies on two different faces in some $(k+1)$-face imbedding. The graph $G-e$ is connected, for otherwise $e$ would lie on only one face, and it has a $k$-face imbedding when clge $e$ is deleted from the $(k+1)$-face imbedding of $G$. By the induction hypothesis, the graph $G-e$ has a minimum cotree $C$ with at most $k-1$ unpaired edges. Thus $C+e$ is a minimum cotree of $G$ with at most $k$ unpaired edges.

A Xuong cotree of graph $G$ is any minimum cotrce of $G$ that admits an adjacency matching with number of paired edges maximized (over all minimum cotrees). The number of unpaired edges in such a cotree is denoted $U(G)$.

Although Xuong seemed to be little concerned with algorithms, Theorem 3.5 is essentially contained in [X79a]. Theorem 3.6, which relates maximum genus to deficicncy, is generally regarded as Xuong's main result.

THEOREM 3.5. [X79A] A connected graph $G$ has maximum genus

$$
\gamma_{M}(G)=\frac{\beta(G)-U(G)}{2}
$$




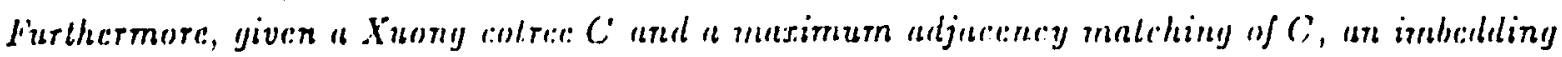
of $G$ that minimizes facccount (and thereby maximizes ycnus. t:an be found in polynomial-time.

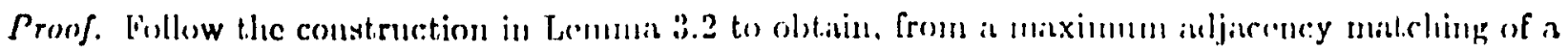

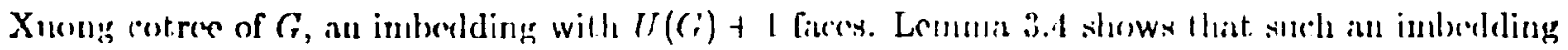

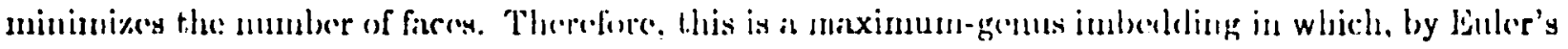
polyludral repuation, $\gamma_{M}(C)=(\beta(C)-U(C)) / 2$.

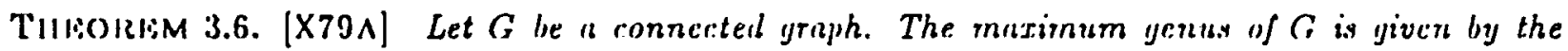
formula

$$
\gamma_{M}(G)=\frac{\beta(G)-\xi(G)}{2}
$$

Proof. Any Xuong cotrec nuust contiain at least aw mnny odd components as $\xi(G)$. Since every odd component must contain at least one unpaired colge, it follows that $U(G) \geq \xi(G)$. Conversoly, a nuaxiunum adjacency matching in the complement $C$ of a Xilung tree must leave at leisst $U(G)$ edges unpaired. The cven conponents of $C$ are completely pairable, and the ordd components are pairable except for one edge left over, thercfore it follows that $\xi(G) \geq U(G)$. Thus $\xi(G)=U(G)$, from which Xuong's equation followy. Morcover, we see that Xuong trecs and Xuong cotrcey, as defined here, are indced complementary objects.

\section{Reduction of Maximum Genus to Linear Matroid Parity}

In order to determine the miximum genus and find a maximum imberlling for an arbitrary graph $G$ in polynomial-time, we have shown that it suffices to slow how to find a Xuong cotree and a maximum adjacency matching of its edges in polynomial time. This problem resembles what is known as the matroid parity problem for cograplic matroids. We use the detinitions relating to matroid parity that are found in Stallman and Gabow's paper on linear matroid parity [SG].

A matroid $M=(E, I)$ consists of a finite ground set $E$ and a family $I$ of "independent" subsets of $E$ satisfying the following axioms:

1. If $A \in I$ and $B \subseteq A$, then $B \in I$.

2. If $A, B \in I$ and $|A|=|B|+1$, then there exists $a \in A$ such that $B+a \in I$. The matroid parity problem [La71] is the following. Given a matroid $M=(E, I)$ and a perfect pairing of the elements of the grouncl set $E$, find an optimum subset of $E$ such that an element is in the subset if and only if its paired edge is in the subset. Optimum means cither a largest subset (the cardinality parity problem) or a maximum weighted subset (the weighted parity problem). Both can be solved in polynomial time for a large class of matroids known as linear (or matric) matroids 


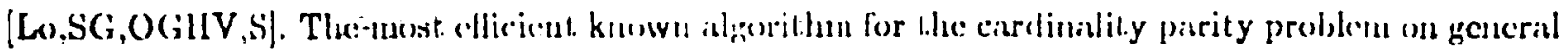

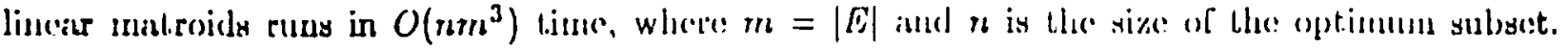
Matroid parity is a gencralization of two well-known problonus: priph matching and matroid intersection.

For any graph $G=(V, E)$, there is a linciar matroid $M=(E, I)$, called the cographice matroid, in which the ground set is the celge sett of the graph and $C \subseteq E$ is an independent sot if and only if $G-C$ is conueterl. Maximun independent sets in cographic matroids are minimum cotrecs of the corresponding graph. For any perfect matching of the edges of the griph, we have an instance of the matroid parity problem on cogriphic matroids, which we call the cotree parily problem. The cardinality parity problems for both griphic (spauning trec) and cographic matroids arc eawier than general lincar matroid parity, and cau be solverl in $O\left(\mathrm{~nm}^{2}\right)$ time [La76,SG]. Stallman and Gabow conjecture that this time bound can be reduced to $O(m n \log n)$.

If each edge of a graph $G$ were adjacent to exictly one other edge, then we could directly apply an algorithm for cotree parity to graph $G$. However, adjaccncy is not an unambiguous pairing rule for most graphs. Therefore, in this section, we shall transform $G$ into an auxiliary graph $G^{\prime}$ with unambiguous pairs. The auxiliary graph $G^{\prime}$ is a subdivision of the graph $G$ itself. Precisely, each edge of $G$ is subdivided into as many elges as its number of edge-neighbors in $G$. Figure 4.1 illustrates such a subdivision.
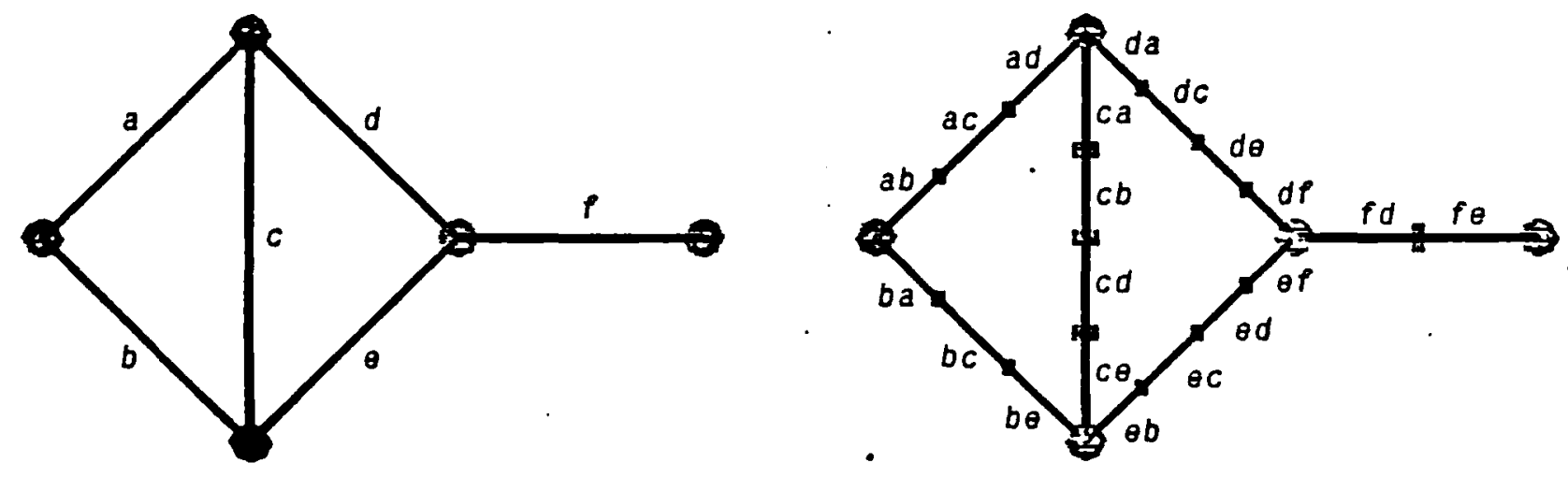

Figure 4.1. A graph $G$ and a corresponding auxiliary graph $G^{\prime}$.

As illustrated in Figure 4.1, we label each edge of the subdivided graph $G^{\prime}$ by a label of the form $x y$, where $x$ is the name of the edge in $G$ of which it is a segment and where $y$ is the name of some distinct neighbor of edge $I$ in the original graph $G$. The choice of which segment of $G$ is to be labeled $x y$, for any particular adjacent edge $y$, is completely arbitrary, provided there is exactly 
one schincut of $x$ labbeled $x y$.

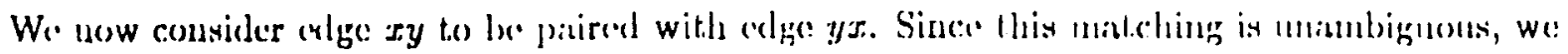
can ibpply a cotre: parity algorithm to $C^{\prime}$ and construct. in polynomial time a minimum cotrece C' with a maximum number of paired crlycy.

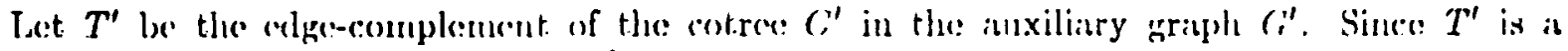
spaming tree for the anxiliary graph $C^{\prime}$, it contains either all the secoments on all but oue of the segments of every colge of the original graph $G$. We now assoriate with spanning tree $T^{\prime \prime}$ in graph $G^{\prime}$ a subgraph $T$ in $G$, according to the rule that an colge $x$ of $G$ apperars in $T$ if and only if every segment of $x$ in $G^{\prime}$ occurs in $T^{\prime}$. It is a consc(puenes of the construction of $G^{\prime}, T^{\prime \prime}$ and $T$ that $T$ is a spanning tree for $G$ : $T$ is acyclic and comnceted because $T^{\prime}$ is acyclic and connected.

Let the elge-complement of spinuing trec $T$ in the original graph $G$ be called $C$. Then $C$ is a minimum cotrce. Two edges of $C$ are matched if and only if they have matched segments in the cotree $C^{\prime}$ of the auxiliary graph $G^{\prime}$.

This adjacency matching of the edges of cotres $C$ in $G$ is a maximum matching among all possible minimum cotrecs of $G$, beciuse there is a bijection from minimum cotrecs of $G$ to minimum cotres of $G^{\prime}$ such that the size of the maximum abljacency matching in the cotree of $G$ exuals the size of the maximum labcled-edge pairing in $G^{\prime}$.

Thus, we have constructed a Xuong cotree for $G$ and a maximum adjacency pairing of its edges in polynomial time.

\section{The Algorithm}

We now summarize and analyze the algorithm for-obtaining a maximum-genus imbedding. Suppose graph $G$ has $v$ vertices, $e$ cdges, and maximum degree $d$. The following steps are uscd.

1. Create auxiliary graph $G^{\prime}$ by subdividing edges in $G$. The new graph has $e^{\prime}=O(e d)$ edges and $v^{\prime}=O(e d)$ vertices. This stcp runs in time $O(e d)$.

2. Run the cotree parity algorithm on graph $G^{\prime}$. Producing a maximum set of paired cotrce edges requires at most $O\left(e^{\prime}\left(v^{\prime}\right)^{2}\right)=O\left(e^{3} d^{3}\right)$ time by the Stallman-Gabow algorithin [SG]. The edge set can be extended to a full minimum cotree by greedily adding unpaired edges. This requires $O\left(\left(e^{\prime}\right)^{2}\right)=O\left(e^{2} d^{2}\right)$ time.

3. For each cotree edge in $G^{\prime}$, label the corresponding edge in $G$ as a cotree cdge. Pair the edges in $G$ which correspond to paired edges in $G^{\prime}$. This requires $O\left(e^{\prime}\right)=O(e d)$ time.

4. Find a one-face imbedding of the spanning tree edges of $G$. This requires $O(e)$ time.

5. Add the paired cotrce edges to the imbedding. The first edge of each pair can be added in 


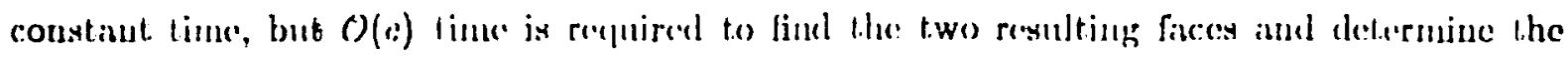

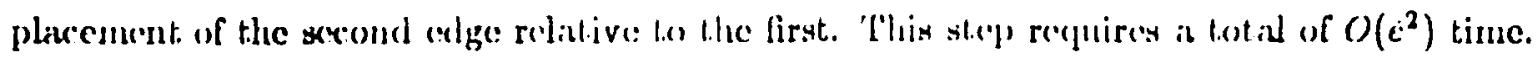

6. Adel unpaired cotres alges to the imbeldiug. This takes $O(v)$ time, since there is at most one: uupieired clge per vertex.

The culies algorithu takes $O\left(e^{3} d^{3}\right)$ time. We use the cotrece parity algorithm on a sperial class of graphs containing many vertices of dengree two, henes the aw tulal time complexity of lhis algoritlum may bre lower. Furthermore, Stallman and Cabow [SG] eomjecture that the actual time complexity of their algorithm for gencral cetree parity is $O(e v \log v)$, which would imply a $O\left(e^{2} d^{2} \log c d\right)$ time bound on our minxinum-gemus algorithn.

\section{Open Problems}

1. The fact that maximuun genus is reducible to linear matroid parity, which is a gencralization of maximum matching, suggests that the corresponding counting problem may be provably difficult. Is it possible that counting the number of ways a graph may be imbedded in a surface of maximum genus is \#P-complete?

2. Our algorithn for computing a maximum genus imbedding runs in time polynomial in the size of the graph. This is the only algorithm we know of for constructing any kind of imbedding that muns in time indepcnclent of the genus. Is it possible to extend the algorithm to return imbeddings in which the genus is a fixed constant less than the maximum?

3. Reif [R79] showed that determining whether a partial imbedding of a graph can be extended to a full imbedding in the same surface is NP-complete. Is the same true for determining whether a partial imbedding can be extended to a one-face imbedding?

4. Suppose graphs $G$ and $H$ are non-isomorphic. One might ask how the non-isomorphism shows up in the way the graphs may be imbedded in different surfaces. Knowing all the "counting information" about how a graph imbeds in all surfaces is not a complete invariant for isomorphism. It clearly isn't a complete invariant for trees, which only have plamar imbeddings, and we have examples of non-isomorphic, highly connected, graphs such that cointing the number of imbeddings in all surfaces does not distinguish them. However, randomly sampling imbeddings and making estimates of the number of ways different graphs imbed in different surfaces may prove to be an interesting new isomorphism heuristic. 


\section{References}

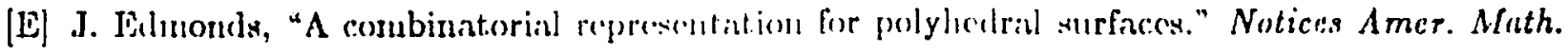
Sisc., 7, 1060, p. 046.

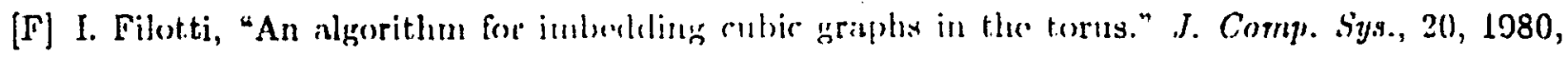
p.). $255-270$.

[FMR] I. Filot.ti, C. Miller, J. Ruif, "On delormining the gromus of in graph in $O\left(v^{O(G)}\right)$ stceps." $11^{\text {th }}$ Annual ACM Symposium on Theory of (omputiny, 1979, pp. 27-37.

[GF] J. Gross, M. Furst, "Hicrarchy for imberlding-distribution inviriants of a graph." Typeseript, 1085.

[GR] J. Gross, R. Rosen, "A linear-time planarity algorithn for 2-complexes." Journal of the ACM, 20, 1979, pp. 611-617.

[GT] J. Gross, T. Tucker, Topolnyical Graph Theory. Wiley-Interscience, to appear.

[H] L. Heffter, "Ü̈er das Problem der Nachbargcbicte." Muth. Ann., 38, 1891, pp. 477-508.

[HT] J. Hopcroft, R. Tarjan, "Efficient planarity tcsting." Journal of the ACM, 21, 1974, pp. 549568.

[J] M. Jungerman, "A characteri\%ation of upper embeddable graphs." Trans. Amer. Math. Soc., 241,1978 , pp. $401-406$.

[La71] E. Lawler, "Matroids with parity conditions: a new class of combinatorial optimization problems.” Electronics Research Laboratory, Bcrkcley. Memo. ERL-M334, 1971.

[La76] E. Lawler, Combinatorial Optimization, Networks, and Mutroids. Holt, Rinehart, and Winston, 1976, 374 pages.

[Lo] L. Lovász, "The matroid matching problem." Algebraic Methods in Graph Theory, for the Colloquia Mathematica Societatis Janos Bolyai, Szeged, Hungary, 1978.

[N] L. Nebesky, "Every connected, locally connected graph is upper embeddable." J. Graph Theory, 5, 1981, pp. 197-199.

[NSW] E. Nordhaus, B. Stewart, A. White, "On the maximum genus of a graph." Joutnal of Combinatorial Theory, Series B, 11, 1971, pp. 258-267.

[OGHV] J. Orlin, E. Gugenheim, J. Hammond, J. VandeVate, "Linear matroid parity made almost easy." Extended abstract. 


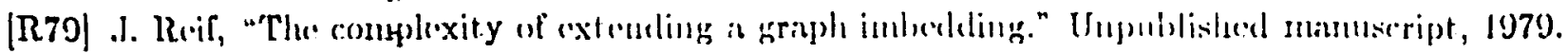

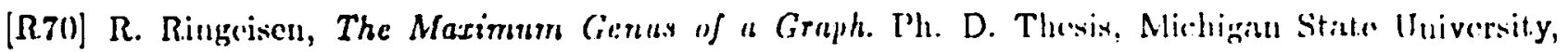
1970.

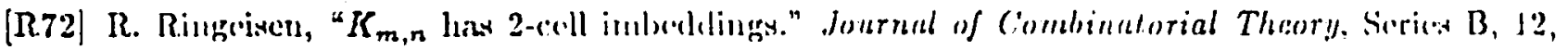
1072, pp. 101-104.

[S] M. Stallınan, "Wcighted matroid parrity." Unpullishod manusscrịt., 1984.

[SG] M. Stallman, H. Gabow, "An angmonting path algorithm for the parity problem on lincis matroids." $25^{\text {th }}$ Symposium on the Foundation.s of Computer Science, 1984. pp. 217-227.

[W] A. Whitc, Graphs, Groups, und Surfaces (2 ${ }^{\text {nd }}$ (rl.). North-IIolliml/Elsevicr Science, 1084, 31.4 pages.

[X79a] N. Xuong, "How to deternine the miximum genus of a griph." Journai of Combinatorial Theory, Scries B, 26, 1079, pp. 216-225.

[X79b] N. Xuong, "Upper-embeddable graphss and related topics." Journal of Combinalorial Theory, Series B, 26, 1979, pp. 226-232.

[Z] J. Zaks, "The maximuin gcuus of cartesian products of graphs." Canad. J. Math., 20, 1974, pp. 1025-1035. 